\title{
Plataforma Virtual para el aprendizaje de la Geometría Analítica
}

Virtual Platform for learning Analytic Geometry

\author{
Verónica Badillo-Torres ${ }^{1}$ \\ Universidad Nacional Autónoma de México \\ Guillermo Rodríguez-Abitia ${ }^{2}$ \\ College of William \& Mary
}

Recibido: 01.09.2021

Aceptado: 30.10.2021

\section{Resumen}

Las ideas se convierten en innovaciónón una vez que se implementan como nuevos productos, servicios o procedimientos y que realmente encuentran una aplicación exitosa en el mercado. Este trabajo está basado en la innovación que se puede generar en el proceso de enseñanza aprendizaje y que se encuentra en la aplicación de una plataforma virtual en la asignatura de Geometría Analítica de la Facultad de Ingeniería de la Universidad Nacional Autónoma de México (UNAM), apoyando el proceso enseñanza-aprendizaje de las Matemáticas. Esta plataforma virtual está basada en las tecnólogaías de información y comunicación (TIC), utilizando el sistema Unity con Moodle, que son accesorios de apoyo de la educación. El objetivo de este trabajo es evaluar si el laboratorio virtual de geometría analítica cumple con la expectativa de ser una innovación efectiva para la educación. Esta herramienta fue creada en 2011 y ha tenido varias versiones, por lo que también se propone hacerle mejoras con base en los resultados de esta evaluación.

\footnotetext{
${ }^{1}$ veronica.badillo@unam.mx ORCID 0000-0003-0812-3876

2 grodriguezabit@wm.edu ORCID 0000-0002-1086-1125
} 
Esta evaluación se llevó a cabo por medio de la aplicación de un cuestionario, resultado de una propuesta adecuada, basada en lo que la literatura existente indica que una plataforma virtual de estas características debe cumplir.

Palabras clave: material didáctico digital, tecnologías de información y comunicación, laboratorio virtual de Geometría Analítica.

\begin{abstract}
Ideas become innovations once they are implemented as new products, services or procedures and they truly find a successful application in the market. This work is based on the innovation that can be generated in the teaching-learning process and that is found in the application of a virtual platform in the subject of Analytic Geometry at the Faculty of Engineering of the National Autonomous University of Mexico (UNAM), supporting the teaching-learning process of Mathematics. This virtual platform is based on information and communication technologies (ICT), using the Unity system with Moodle, which are support accessories for education. The objective of this work is to evaluate whether the virtual laboratory of analytical geometry meets the expectation of being an effective innovation for education. This tool was created in 2011 and has had several versions, so it is also proposed to make improvements based on the results of this evaluation.
\end{abstract}

This evaluation was carried out through the application of a questionnaire, the result of an adequate proposal, based on what the existing literature indicates that a virtual platform of these characteristics must meet.

Keywords: digital didactic material, information and communication technologies, virtual laboratory of Analytical Geometry. 


\section{Introducción}

Los constantes cambios que en todos los ámbitos de la sociedad se han producido en los últimos años, conllevan a una continua actualización en el empleo de las nuevas tecnologías de información y la comunicación (NTIC).

Para Marqués (2000), las NTIC se convierten en una herramienta cada vez más indispensable en las instituciones educativas, donde pueden realizar múltiples funciones incluyendo: fuente de información; canal de comunicación interpersonal, para el trabajo colaborativo y para el intercambio de información e ideas; medio de expresión y para la creación (procesadores de textos y gráficos, editores de páginas web y presentaciones multimedia, cámara de vídeo); instrumento cognitivo y para procesar la información como las hojas de cálculo, gestores de bases de datos, instrumento para la gestión, ya que automatizan diversos trabajos de la gestión de los centros de trabajo; actividades secretariales; acción tutorial; control de asistencias; y bibliotecas y recursos interactivos para el aprendizaje. Los materiales didácticos multimedia informan, entrenan, simulan, guían aprendizajes, motivan y constituyen un medio lúdico y para el desarrollo psicomotor y cognitivo.

Actualmente se está desarrollando en la Coordinación de Matemáticas de la División de Ciencias Básicas (DCB) de la Facultad de Ingeniería de la UNAM, material didáctico digital como apoyo en el proceso enseñanza-aprendizaje de las matemáticas, que permita al estudiante trabajar de una manera interactiva y visual, para reforzar conceptos que los lleve a un aprendizaje significativo.

Se ha detectado que, en general, a los alumnos que cursan la asignatura de Geometría Analítica les es complicado el paso de trabajar en un espacio de dos dimensiones a trabajar en un espacio de tres dimensiones. Sumando los bajos índices de aprobación que se han venido presentando en dicha asignatura, surge la necesidad de apoyar a los alumnos en este proceso de aprendizaje. 
Para apoyar a los estudiantes que cursan la asignatura antes mencionada, se ha estado elaborando un material didáctico digital, que les permita a los estudiantes una mejor visualización en el espacio tridimensional, reforzar los conceptos a través de prácticas en el laboratorio virtual de Geometría Analítica e inducir a una formación científica.

\section{Marco teórico}

\subsection{Las tecnologías de información y comunicación (TIC)}

A partir de la segunda mitad del siglo XX, la enseñanza compuesta de la ciencia con la tecnología se ha intensificado. La incidencia de las tecnologías de la información y la comunicación (TIC) en los diferentes ámbitos sociales es cada vez más notable, las posibilidades de acceso a la información y las herramientas que posibilitan la gestión de la misma son cada vez mayores y diversas, lo cual conlleva la necesidad de formar ciudadanos que, adicionalmente a los conocimientos específicos de su área, tengan las competencias digitales necesarias para la utilización de dichas herramientas.

En la opinión de Gracia (2005) la educación no es otra cosa que el trabajo que cada uno pone inconsciente o conscientemente, ayudado de un modo indirecto o intencional por otros, y siempre bajo las influencias del medio en que vive, para realizarlo con el nivel de perfección de que sea susceptible, y de las exigencias del entorno y el objetivo.

Por otra parte, Romero y Sarmiento (2012) citan que la educación es un proceso social mediante el cual se asimila a los diferentes miembros, según sus propias reglas, valores, ideologías, prácticas, proyectos y saberes compartidos por la mayoría de la sociedad.

Las TIC son algo más que informática y computadoras, puesto que no funcionan como sistemas aislados, sino en conexión con otras mediante una red. También son más que tecnología de emisión y difusión (como televisión y radio), puesto que no sólo dan cuenta de 
la divulgación de la información, sino que además permite una comunicación interactiva del proceso.

El uso de la tecnología puede llegar a ser una herramienta poderosa para que los estudiantes logren crear diferentes representaciones de ciertas tareas y sirve como un medio para que formulen sus propias preguntas o problemas, lo que constituye un importante aspecto en el aprendizaje de las matemáticas (Barrera y Santos, 2001).

La incorporación de las TIC en el ámbito educativo obedece a que actúan como impulsoras para la innovación educativa cambiando los métodos tradicionales de enseñanza. Utilizadas adecuadamente, pueden proporcionan apoyo para lograr mejores aprendizajes, adquiriendo conocimiento y en cierta forma a reducir el fracaso escolar. Así, contribuyen a hacer más eficientes los procesos de enseñanza y de aprendizaje.

Para Sánchez (2009). una plataforma de enseñanza virtual es un sistema de educación a través de Internet, en donde se encuentran las siguientes denominaciones:

. Virtual learning environment (VLE) - Entorno virtual de aprendizaje.

. Learning management system (LMS) - Sistemas de gestión del aprendizaje.

. Course management system (CMS) - Sistema de gestión de cursos.

. Managed learning environment (MLE) - Ambiente administrado de aprendizaje.

. Integrated learning system (ILS) - Sistema integrado de aprendizaje.

. Learning support system (LSS) - Sistema soporte al aprendizaje.

. Learning platform (LP) - Plataforma de aprendizaje.

El e-learning consiste de la educación y capacitación a través de Internet, es decir el tipo de enseñanza online que permite la interacción del usuario con el material mediante la utilización de diversas herramientas informáticas. 
El e-learning comprende fundamentalmente los siguientes aspectos:

. El pedagógico, es decir que la tecnología educativa como disciplina de las ciencias de la educación, está ligada a los medios tecnológicos, la psicología educativa y la didáctica.

- El tecnológico, referido a la tecnología de información y comunicación, mediante la selección, diseño, personalización, implementación, alojamiento y mantenimiento de soluciones en dónde se integran tecnologías propietarias.

. El apoyo a las asignaturas presenciales o llamado el término blended learning, que consiste en combinar la formación presencial con la formación a través de las tecnologías de información y comunicación, tratando de aprovechar todas las ventajas de éstas en los procesos de aprendizaje presenciales.

\subsection{La Geometría Analítica}

Gracias a los estudios y ensayos propuestos por René Descartes y Pierre de Fermat, se generaron los fundamentos de la geometría analítica, en donde se estudia las figuras geométricas mediante técnicas, así como la correspondencia entre una ecuación y el lugar de las coordenadas que satisfacen la ecuación.

Con respecto a la comprensión propia de los objetos geométricos, el National Council of Teacher of Mathematics (NCTM) (2000) destaca que es con el aprendizaje de esta disciplina que los estudiantes aprenderán sobre las características y relaciones de estos, así como la construcción y manipulación mental de las diferentes representaciones de objetos en dos y tres dimensiones.

Asimismo, el NCTM señala que la geometría constituye un terreno fértil para el desarrollo de las habilidades para generar razonamiento y justificación. 
Las investigaciones de Moreno (2002), Duval (1998), Herscowitz y Vinneer (1987) reconocen que el aprendizaje de la geometría es un proceso complejo, debido al uso de las representaciones visuales de conceptos y procesos de otras áreas en matemáticas y en otras ciencias, constituye un punto de encuentro de una matemática teórica y una que genera modelos, y brinda un significado a el objeto y propiedades geométricas.

\subsection{La plataforma virtual (TICS-Geometría Analítica)}

De acuerdo con Gutiérrez (2005) el uso de las TIC en la enseñanza de las matemáticas sugiere el uso de software de geometría dinámica, pues permite a los estudiantes la exploración y verificación de propiedades geométricas, así como la automatización del cálculo geométrico.

La naturaleza constructivista del procesador geométrico desarrolla en los estudiantes aptitudes para realizar en poco tiempo la tarea encomendada y proceder a explorar otras posibilidades.

Por su parte, Cuevas (1999) menciona que las transformaciones que la humanidad ha experimentado en las últimas décadas constituyen un reto para la pedagogía de las matemáticas en el nivel universitario. Se imponen transformaciones en los métodos y medios de enseñanza que tradicionalmente se han venido empleando. Además, la computadora se ha insertado al proceso de enseñanza y aprendizaje para enriquecerlo. Su uso adecuado incrementará la eficiencia y calidad del mismo.

Las transformaciones que se han producido en los procesos formativos provocados por la creación de los nuevos entornos virtuales de aprendizaje mediados por las tecnologías de información y comunicación (TIC), están contribuyendo a definir nuevos roles y funciones entre los implicados (Del Moral, 1998a), e incluso a construir escenarios de aprendizaje abiertos exentos de las limitaciones de la enseñanza tradicional. Así los alumnos pasan a ser los máximos responsables de sus aprendizajes, construyendo su propio conocimiento a partir de las diversas experiencias recogidas por la multiplicidad de medios. Los nuevos canales de 
comunicación están dando cabida a multitud de informaciones, y desarrollando destrezas, relacionadas en gran medida con participaciones en línea y favoreciendo las habilidades de búsqueda que ya son imprescindibles en estos espacios abiertos, favorecidos por la red Internet (Solano, 1998). Todo ello implica una nueva forma de construcción del conocimiento y de generar el aprendizaje.

Marqués (2009), señala cinco dimensiones de la competencia digital:

. Dimensión del aprendizaje, que abarca la transformación de la información en conocimiento y su adquisición.

. La dimensión informacional, que abarca la obtención, la evaluación y el tratamiento de la información en entornos digitales.

. La dimensión comunicativa, que abarca la comunicación interpersonal y la social.

. La dimensión de la cultura digital, que abarca las prácticas sociales y culturales de la sociedad del conocimiento y la ciudadanía digital y

. La dimensión tecnológica, que abarca la alfabetización tecnológica, el conocimiento y dominio de los entornos digitales, siendo las capacidades asociadas: (a) aprender y generar conocimientos, productos o procesos, (b) obtener, evaluar y organizar información en formatos digitales, (c) comunicarse, relacionarse y colaborar en entornos digitales, (d) actuar de forma responsable, segura y cívica y (e) utilizar y gestionar dispositivos y entornos de trabajo digitales.

Para Silvio (2006) la virtualización nos da un proceso y resultado del tratamiento y comunicación mediante el uso de la computadora, informaciones y conocimientos, así como los elementos cuya manipulación permite realizar diferentes operaciones como aprender en un programa, consultar documentos, comunicarse entre alumnos, profesores y otros. 
Para Navarro (2000), los recursos en educación se definen como maneras o herramientas que habilitan las funciones e instrucciones.

Existen diferentes marcos de referencias como el de Ardila-Rodríguez (2011) y Torres (2003), donde se describen los procesos propios que caracterizan la información en los ambientes virtuales incluyendo:

- Proceso: funcionamiento del curso, planificación y programación. En este apartado las plataformas deben de contener el desarrollo o la puesta en ejecución del curso. En donde se tome indicadores base para la evaluación de las actividades de enseñanza-aprendizaje y donde se concreta en los objetivos y la programación de actividades de enseñanza y aprendizaje

. Proceso: contenidos programáticos de enseñanza. En este apartado se mide la calidad de los contenidos disponibles para el estudio, para lograr un avance significativo en el aprendizaje.

. Proceso: comunicación e interacción. Es importante que los alumnos y el profesor se pueda logra una comunicación e interacción por lograr un aprendizaje.

Tomando en cuenta los anteriores procesos descritos anteriormente se propone un cuestionario basado en ellos.

\subsection{Desarrollo de material didáctico virtual.}

Desde hace varios años, se ha venido desarrollando material didáctico digital en la Facultad de Ingeniería UNAM, como apoyo al proceso enseñanza-aprendizaje en Geometría Analítica, que permita al estudiante trabajar de una manera interactiva y visual, para reforzar conceptos que los lleve a un aprendizaje significativo, llamado Laboratorio Virtual de Geometría Analítica (LVGA) 
El laboratorio consta de facilitar la interacción de los alumnos con objetos geométricos, a través del uso de avatares en ambientes tridimensionales, guiados por actividades planeadas de aprendizaje. La herramienta se ilustra en la figura 1.

Figura 1. Imagen de la actividad en el LVGA.

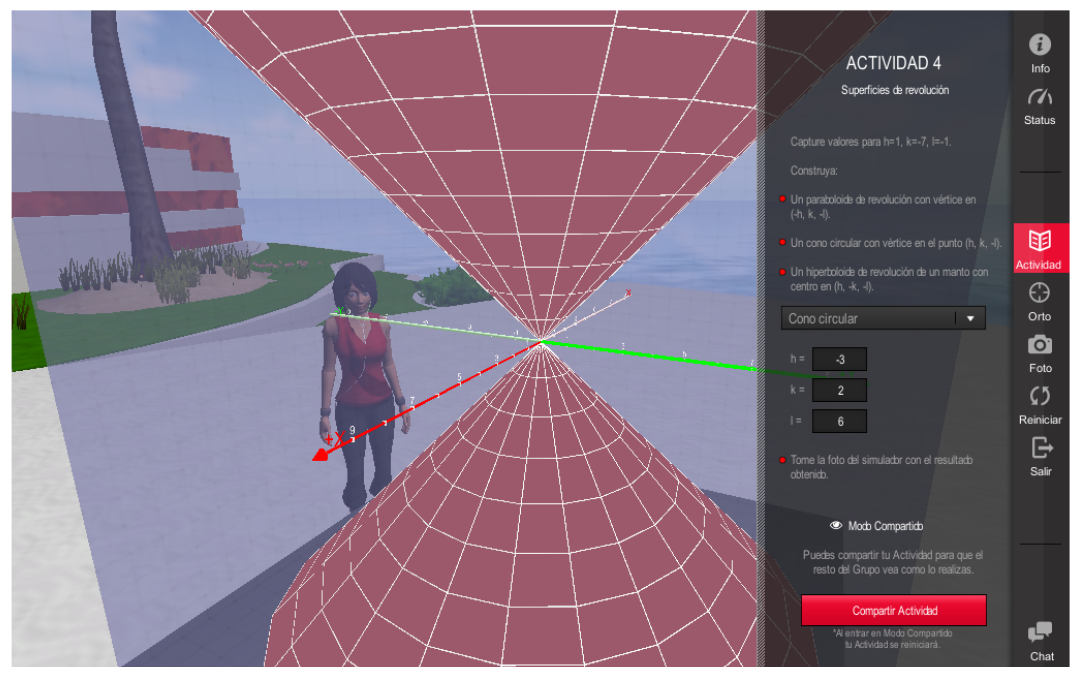

Fuente: elaboración propia.

\section{Metodología}

Para evaluar el LVGA se desarrolló un cuestionario que incluye los procesos identificados en la literatura.

Las preguntas están agrupadas de acuerdo con el proceso que abordan, como se indica a continuación:

\section{Proceso: asignación y utilización de medios y recursos}

1. ¿En la página de laboratorio virtual de Geometría Analítica se encuentran establecidos los objetivos de los temas a realizar?

2. ¿Se asigna el tiempo requerido para cada actividad? 
3. ¿El ritmo (avance) del curso en el laboratorio virtual le parece apropiado?

4. ¿Cree usted que los materiales están adaptados a las condiciones del ambiente virtual? Proceso: contenidos programáticos de enseñanza

5. ¿Se adecúa la práctica a los contenidos?

6. ¿Los contenidos del laboratorio virtual logran los objetivos de la asignatura?

Proceso: comunicación e interacción

7. ¿Se motiva a los estudiantes a comunicarse con el docente?

8. ¿El sistema permite a los estudiantes compartir ideas y conocimientos?

Proceso: asignación y utilización de medios y recursos

9. ¿Qué tan fácil es el manejo de la interfaz de la plataforma?

10. ¿Se incorporan materiales bibliográficos multimedia al curso biblioteca virtual?

11. ¿Hay actividades de autoevaluación del aprendizaje?

La anterior encuesta propuesta fue aplicada a 4 grupos mismos que asigno la Coordinación de Matemáticas de la División de Ciencias Básicas. De los cuatro grupos, 2 fueron intervenidos con la utilización del laboratorio virtual, mientras que otros dos fungieron como grupos de control utilizando una metodología tradicional de enseñanza. Los grupos estaban compuestos de la siguiente manera:

. Grupo 04 con un total de 37 alumnos, con intervención

. Grupo 22 con un total de 42 alumnos, como control

. Grupo 24 con un total de 19 alumnos, con intervención

. Grupo 28 con un total de 40 alumnos, como control

La encuesta fue aplicada a 138 alumnos, siendo 45 mujeres y 93 hombres, cuyas edades están distribuidas como se muestra en la figura 2. 
Figura 2. Distribución muestral por edad.

\section{Edad}

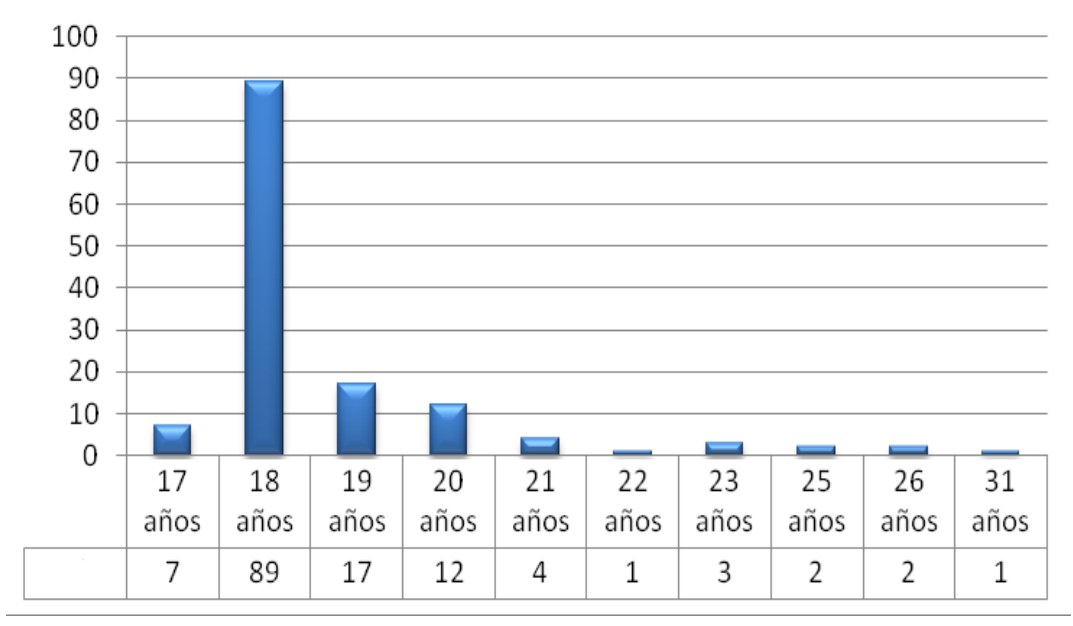

Adicionalmente a la medición de los procesos como resultado de la encuesta, se calcularon los promedios de calificaciones y se calculó la relación entre los procesos y el promedio mediante regresión lineal.

Además, los estudiantes participantes, al tratarse de una materia de tronco común en los programas de estudio, podían ser de diferentes carreras dentro del área de ingeniería. La distribución por especialidad se muestra en la figura 3.

Figura 3. Distribución muestral por especialidad de estudios.

\section{Ingeniería}

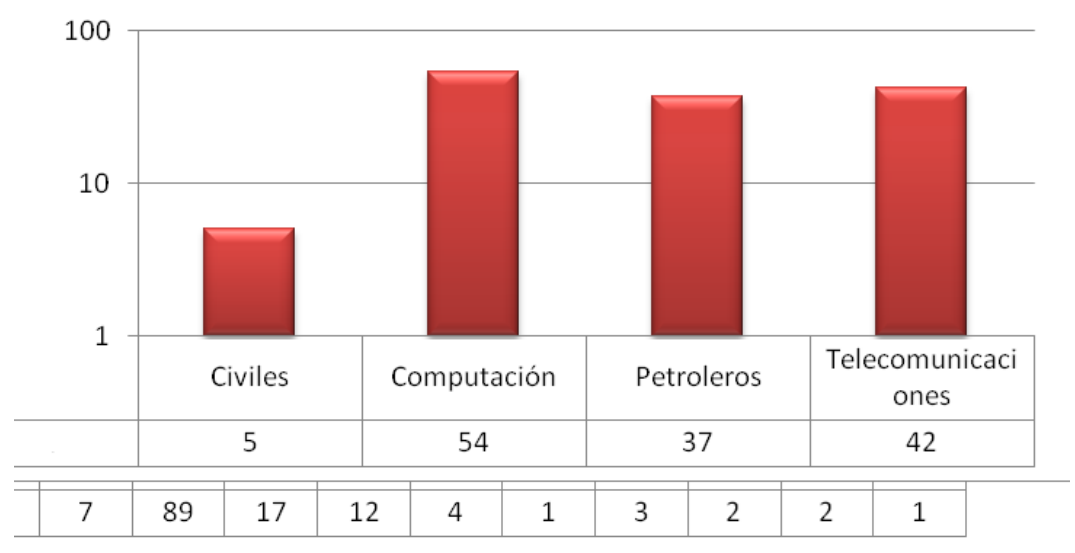




\section{Resultados}

En los grupos 04, 22, 24 y 28 se obtuvieron un promedio por proceso y su promedio de 3 actividades o prácticas generando una gráfica de tendencia (regresión) por cada proceso representada por una línea de diferente color.

La figura 4 presenta el comparativo de los resultados para cada uno de los cuatro grupos analizados.

Figura 4. Grafica comparativa de los cuatro grupos.

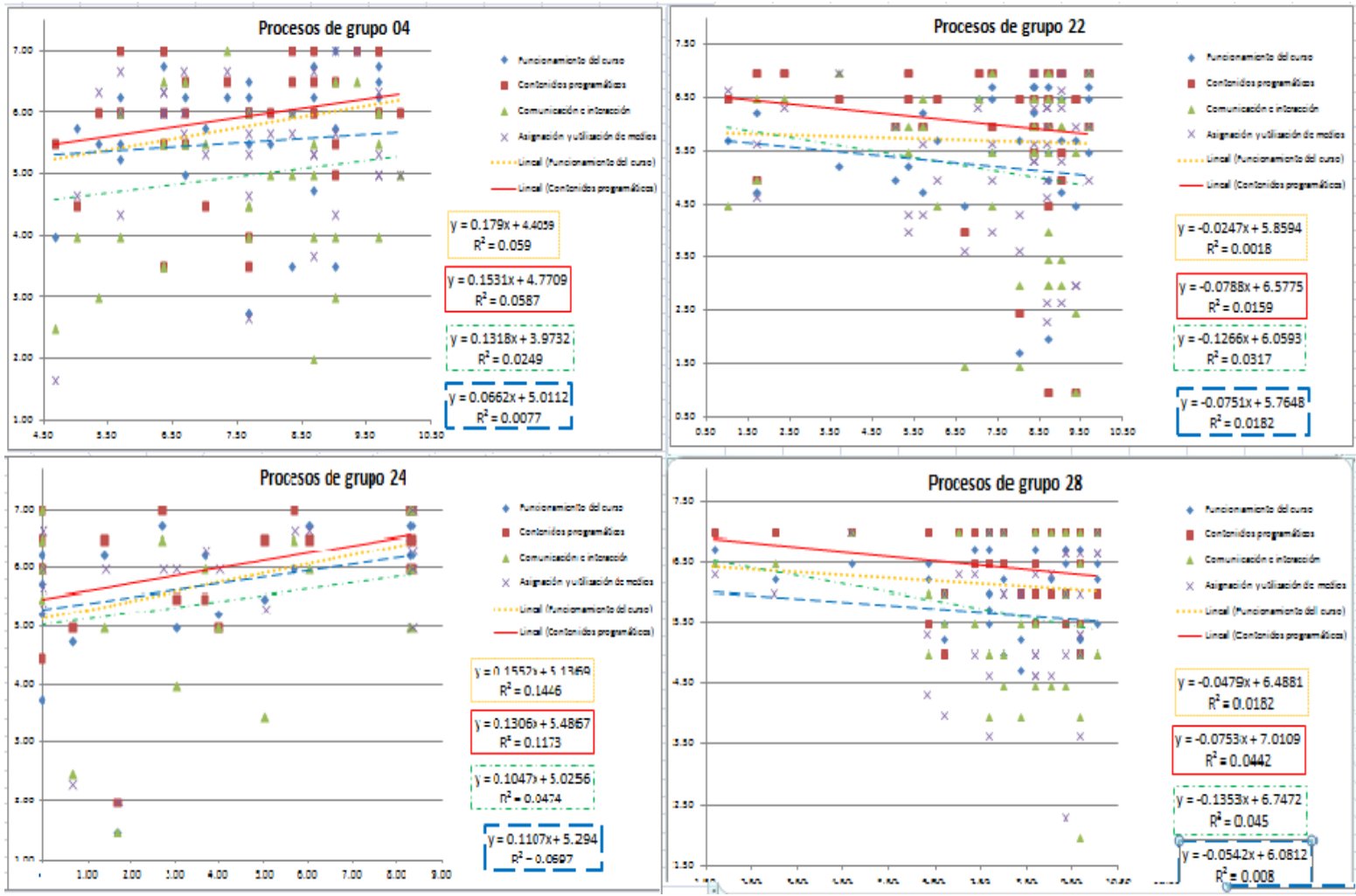

Las gráficas muestran los grupos 04 y 24, es decir aquellos donde hubo intervención con el laboratorio virtual, resultan en pendientes positivas, indicando que el LVGA les ayudo en el aprendizaje, pero los otros dos grupos reportan pendientes negativas indicando que los medios de apoyo utilizados no resultaron efectivos para apoyar el aprendizaje significativo, o al menos el resultado. 
En condiciones normales, el índice de aprobación de la asignatura no supera el 50\%. En este ejercicio, se logró un porcentaje de eficiencia superior al 75\%, sugiriendo el gran potencial de aplicabilidad de la herramienta.

\section{Conclusiones}

Si bien existen otros factores de explicación alternativa a los resultados planteados, como puede ser la calidad de los profesores en cada grupo, la capacidad de los mismos para utilizar la herramienta y su nivel de comodidad con la aplicación de habilidades digitales, o la calidad de las actividades planeadas alrededor de la simulación de realidad virtual, es cierto que la aplicación de TIC para la enseñanza no es un sistema simple ni meramente técnico.

Como todo sistema sociotécnico, es importante reconocer que cada escenario tiene características propias y que una sola tecnología no cumple con todos los requisitos. La realidad virtual es particularmente útil donde se requiera de un apoyo gráfico que permita conceptualizar lo que es difícil hacer con solo la imaginación. La Geometría Analítica, siendo tridimensional por naturaleza, es un claro ejemplo de gran potencial.

Cada uno de los procesos mencionados debe ser cuidadosamente planeado y ejecutado con el fin de potenciar el beneficio de la innovación que se propone. Solo con una armoniosa combinación de factores se puede aprovechar realmente a la innovación tecnológica en el aula.

\section{Referencias}

Ardila-Rodríguez, M. (2011) Indicadores de calidad de las plataformas educativas digitales. educ.educ. [online]. vol.14, n.1, pp.189-206. ISSN 0123-1294.

CUDI (2006), Modelo y Escenarios para la Integración de OA. Módulo III [En línea], obtenido en marzo de 2011.

http://hosting.udlap.mx/estudiantes/jose.ferrercz/importando_moodle.pdf 
García Alcantara, P. (2005) Compendio de pedagogía teórico-práctica, Biblioteca Virtual Universal, Argentina. http://www.biblioteca.org.ar/libros/89819.pdf

Gamboa, R. (2007) Uso de la tecnología en la enseñanza de las matemáticas, Cuadernos de Investigación y Formación en Educación Matemática. 2(3), pp.11-44.

Marqués, G. (2000), Impacto de las TIC en Educación: Funciones y limitaciones [En línea], obtenido en marzo de 2011. http://www.peremarques.net/siyedu.htm

Ogalde, C. I., González V. M. (2008), Nuevas Tecnologías y Educación. Diseño, desarrollo, uso y evaluación de materiales didácticos, México, D.F., $1^{\text {a }}$. Edición. Ed. Trillas.

Romero Sánchez, J. J.y Sarmiento Ortiz, N. A. (2012). Las Tecnologías de la Información y la Comunicación (TIC) como herramientas de apoyo para los procesos de mediación en la asignatura de métodos numéricos aplicados a la ingeniería. Bucaramanga. http://repositorio.uis.edu.co/jspui/bitstream/123456789/2258/2/142937.pdf

Sánchez J. (2013) Plataformas de Enseñanza Virtual para Entornos Educativos. http://www.sav.us.es/pixelbit/pixelbit/articulos/n34/15.pdf 
REVISTA INTERNACIONAL DE PEDAGOGÍA E INNOVACIÓN EDUCATIVA

Volumen 2. Número 1. Enero - Junio 2022 ISSN: 2745-0341 (En línea) 